\title{
On multicollinearity in production functions with collinear inputs and correctly specified disturbances
}

\author{
Isaac D. Essi, N. M. Nafo and E. H. Amadi \\ Department of Mathematics/Computer Science, Rivers State University of Science \& \\ Technology, Port Harcourt, Nigeria.
}

\begin{abstract}
The Cobb-Douglas model is widely applied in econometrics and other areas of research. The model, with strongly collinear inputs has been estimated under alternative correctly specified error terms. The impact of multicollinearity, is seen to be more severe in the multiplicative error model than in the additive error model.Our result also shows that the trend of the effect of multicollinearity is ambiguous as sample size increases-sample size cannot be said to have purged the models of the effect of multicollinearity.
\end{abstract}

Keywords: Cobb-Douglass model, multiplicative error model, additive error model, multicollinearity

\section{INTRODUCTION}

As observed by econometricians and scientists in other disciplines the $p$ - input variable function:

$$
\mathbf{f}=\theta_{0} \prod_{i=1}^{p} X_{i}^{\theta_{i}}
$$

plays a significant role in modeling certain phenomena. In economics, for instance, it is widely applied in research works on production, demand (including transport demand) and cost functions. In biometry, the function (1.1) may be used in carrying out leaf rectangularity index analysis as in [ 3 ] and [5]. In economics, the powers of the X's are called elasticity's and their sum is interpreted as a measure of returns to scale. Each $\theta_{i}(i=1,2, \ldots, p)$ under some conditions gives the factor share of the associated input variable $\mathrm{X}_{i}$.

Econometric model demands the incorporation of an error term as well as the specification of its distribution. The specification of the error term is a major problem in applied econometrics. The functional form $f$ in Equation (1.1) cannot be decided in isolation from the specification of the error term. Since economic theory cannot give precisely always what the functional form should be , as cited in [1] and [ 14 ], this and the related problem of error specification may be resolved empirically.

\section{Theoretical framework and literature review}

\section{Assumption 2.1}

Let us assume a sequence of real valued responses $y_{t}$ with the form

$$
\mathrm{y}_{\mathrm{t}}=\mathrm{f}_{\mathrm{t}}(\theta)+\mathrm{u}_{\mathrm{t}}, \mathrm{t}=1,2, ., \mathrm{T}
$$

where $f_{t}=f\left(X_{t}, \theta\right)$ are known continuous functions on a compact subset $\Theta$ of the Euclidean space $I^{p}$ and the $u_{t}$ are independently and identically distributed errors with zero mean and finite variance $\sigma^{2}>0$. The values of $\theta$ and $\sigma^{2}$ are unknown but fixed. $X_{t}$ is an input vector for the period $t$ and is fixed.

Definition 2.1 (Least Square Estimate)

Any vector $\hat{\theta}$ in $\Theta$ which minimizes

$\mathrm{Q}_{\mathrm{T}}(\theta)=\frac{1}{\mathrm{~T}} \sum_{\mathrm{i}=1}^{\mathrm{T}}\left(\mathrm{y}_{\mathrm{t}}-\mathrm{f}_{\mathrm{t}}(\theta)\right)^{2}$

is called a least square estimate of $\theta$ based on the first $T$ values of $\mathrm{y}_{\mathrm{t}}$.

Theorem 2.1 (Minimum Mean Square Error Criterion (See [ 2 ])

a) Let

Ho: $\quad y_{t}=f_{t}\left(x_{t}, \theta\right)+u_{o t}, t=1,2, \ldots T$

be the model that is to be used to estimate $\theta$, where $f_{t}\left(X_{t}, \theta\right)$ is a known continuous function on a compact subset $\Theta$ of a p-dimensional Euclidean space $\mathrm{IR}^{\mathrm{p}}$ and the $\mathrm{u}_{\mathrm{ot}}$ are identically distributed errors with zero mean and finite various $\sigma^{2}>0$.

b) Let

$\mathrm{H}_{1}: \quad \mathrm{y}=\mathrm{G}\left(\mathrm{Z}_{\mathrm{t}}, \lambda\right)$ 
be an alternative structure that may be used to explain y as well, where $G\left(Z_{t}, \lambda\right)$ embodies both the deterministic and stochastic parts of $y_{t}$ and the stochastic disturbance term may be additive or multiplicative.

c) The function $f_{t}\left(X_{1}, \theta\right)$ and the deterministic part of the model $\mathrm{H}_{1}$ may be linear or nonlinear.

d) Let the estimated adjusted $\bar{R}^{2}$ for $\mathrm{H}_{\circ}$ and $\mathrm{H}_{1}$ be respectively $\overline{\hat{R}}_{0}^{2}$ and $\frac{\wedge^{2}}{R}$. If Ho is the correct model such that $\mathrm{E}\left(\stackrel{\wedge}{R}_{0}^{2}\right)=\bar{R}_{0}^{2}=1$, Then,

$\operatorname{MSE}\left({\overline{R_{0}}}_{0}^{2}\right) \leq \operatorname{MSE}\left(\hat{\bar{R}}_{1}^{2}\right)$.

implies that $\quad \stackrel{\wedge}{R}_{0}^{2} \geq \bar{\wedge}^{2}$

on the overage, where $\operatorname{MSE}\left(\stackrel{\wedge}{R}_{0}^{2}\right)$ is the mean square error of $\frac{\wedge^{2}}{R_{0}}$.

MSE $\left(\hat{R}_{1}^{2}\right)$ is similarly defined.

Proof: The proof can be seen in [2, 7 ].

We shall use this criterion later in comparing two competing models

The minimum variance criterion of [ 12 ] is replaced by minimum mean square criterion of Essi in [ 2 ]. The Essi criterion, which is in terms of the mean squared error (MSE) of the adjusted coefficient of determination is more preferred, for some obvious reasons, to that of Theil, as a basis for comparing estimates of the true and mis-specified models, especially where we use simulated models with replications. In using Theil minimum variance criterion, the response variables in both $\mathrm{Ho}$ and $\mathrm{H}_{1}$ must be in the same units and the models should be

linear. The use of MSE $\left(\hat{R}^{2}\right)$ overcomes these drawbacks. We also benefit as the ratio of MSE $\stackrel{\wedge}{R}^{2}$

$(\bar{R})$ from two competing models, can be used to assess the overall relative efficiency of a set of one model estimates to that of another.

The consequences of an incorrect form for the disturbance term, according to [ 10 ] are bias and inconsistency in the least square estimate of the parameters. Suppose that the error term is correctly specified, be it additive error or multiplicative error, what happens? Heben in [11 ] observes that there is trouble with the multiplicative error model (MEM) in that one may " encounter severe multicollinearity between $\mathrm{K}$ and $\mathrm{L}$, especially with cross-section (rather than time - series) data and especially if our observations are for firms in a fairly homogenous industry". This, Heben says, is " because for such an industry, the capital-labour mix is fairly uniform across firms, since all use more or less the same technique, hence if the $\mathrm{K} / \mathrm{L}$ ratio is, say, 3 , then for all observations we would have approximately $\mathrm{K}=3 \mathrm{~L}$, and hence very strong collinearity."

Fabrycy in [ 8 ] observes that " using linear least squares regressions induce us to adopt functions which are linear in parameters. Often this imposes unrealistically rigid constraints which may create multicollinearity. Using more realistic nonlinear forms and nonlinear least squares regressions is likely to overcome this problem." The papers [ 4 ] and [ 7 ] consider the consequences of mis-specifying the error term for the Cobb-Douglas production model . The articles [ 4 ] and [ 7 ] observe that the consequence is more serious when a multiplicative error plagued data set is fitted with an additive error based model than vice-versa. This trend, it is observed, persists in the presence of multicollinearity.

\section{METHODOLOGY AND DATA}

The two correctly specified models considered are

$$
\text { AED / AEM : } y=\theta_{1} K^{\theta_{2}} L^{\theta_{2}}+U_{0}
$$

and

$$
\text { MED / MEM : } y=\theta_{1} K^{\theta_{2}} L^{\theta_{3}} e^{U_{0}}
$$

The specification AED/AEM is the one where an additive error-plagued data (AED) is fitted with an additive error-based model (AEM). The model MED/MEM is one where a multiplicative errorplagued data (MED) is fitted with a multiplicative error-based model (MEM).

The model (3.2) is intrinsically linear and OLS estimation of the log-transformed version provides estimate for $\left(\theta=\theta_{1}, \theta_{2}, \theta_{3}\right)^{\prime}$. The modified GaussNewton algorithm is used in estimating the intrinsically non-linear model (3.1). The choice of model parameters $\left(\theta_{1}, \theta_{2}, \theta_{3}\right)$ is such that $\theta_{2}+\theta_{3}<1, \theta_{2}+\theta_{3}=1$ and $\theta_{2}+\theta_{3}>1$ but 
Am. J. Sci. Ind. Res., 2011, 2(4): 588-591

$\theta_{2}+\theta_{3}<2$ while the value of $\theta_{1}$ is arbitrary and kept at $\theta=10.0$. We use as our set of parameters $\left(\theta_{1}, \theta_{2}, \theta_{3}\right)=(10.0,0.45,0.50)$.

The input matrix is made of two variables $\mathrm{K}$ (capital) and $\mathrm{L}$ (Labour) and are randomly generated and normally distributed independent variables such that they are typical of data set on capital and labour as that of [ 13 ]. We adopt the data generating process outlined in [ 4 ] and [ 7 ]

The noisy Y's are obtained according to the relations (3.1) and (3.2). The Monte Carlo study uses sample size of 20,40, 80 with each experiment replicated 20 times under three levels of collinearity between $\mathrm{K}$ and $\mathrm{L}$. The levels of collinearity are furnished by simple Pearson correlation coefficient between $\mathrm{K}$ and $\mathrm{L}$. We denote this coefficient by Cor $(\mathrm{K}, \mathrm{L})$ and the values for this work are $0.03,0.24$ and 0.45 .

Empirical results: Altogether we estimated 360 equations. Some of the numerical results obtained are summarized and presented in Tables 1,2 and 3 below .

Table 1 : Values of $\operatorname{MSE}\left(\hat{\bar{R}}^{2}\right)$ for AEDIAEM ( $\sigma_{0}^{2}=0.16, \mathbf{N}=\mathbf{2 0}$ )

\begin{tabular}{|c|l|l|l|}
\hline $\begin{array}{c}\text { Sample Size } \\
(\mathbf{T})\end{array}$ & $\begin{array}{l}\operatorname{Cor}(\mathrm{K}, \mathrm{L})= \\
\mathbf{0 . 0 3}\end{array}$ & $\begin{array}{c}\operatorname{Cor}(\mathrm{K}, \mathrm{L})= \\
\mathbf{0 . 2 4}\end{array}$ & $\begin{array}{c}\operatorname{Cor}(\mathrm{K}, \mathrm{L})= \\
\mathbf{0 . 4 5}\end{array}$ \\
\hline 20 & $3.60 \mathrm{E}-10$ & $4.36 \mathrm{E}-10$ & $2.81 \mathrm{E}-10$ \\
\hline 40 & $3.40 \mathrm{E}-10$ & $4.25 \mathrm{E}-10$ & $2.41 \mathrm{E}-10$ \\
\hline 80 & $23.29 \mathrm{E}-10$ & $4.09 \mathrm{E}-10$ & $2.60 \mathrm{E}-10$ \\
\hline
\end{tabular}

Table 2 : Values of $\operatorname{MSE}\left(\hat{\wedge}^{2}\right)$ for MED/MEM ( $\sigma_{0}^{2}=0.16, \quad \mathbf{N}=\mathbf{2 0}$ )

\begin{tabular}{|c|l|l|l|}
\hline $\begin{array}{c}\text { Sample } \\
\text { Size (T) }\end{array}$ & $\begin{array}{l}\operatorname{Cor}(\mathrm{K}, \mathrm{L}) \mathbf{=} \\
\mathbf{0 . 0 3}\end{array}$ & $\begin{array}{c}\operatorname{Cor}(\mathrm{K}, \mathrm{L})= \\
\mathbf{0 . 2 4}\end{array}$ & $\begin{array}{c}\operatorname{Cor}(\mathrm{K}, \mathrm{L})= \\
\mathbf{0 . 4 5}\end{array}$ \\
\hline 20 & 0.046419 & 0.050798 & 0.035785 \\
\hline 40 & 0.0546240 & 0.031281871 & 0.03304124 \\
\hline 80 & 0.044565 & 0.029809 & 0.032017 \\
\hline
\end{tabular}

Table 3 : Ratio of $\operatorname{MSE}\left(\hat{R}^{2}\right)$ in MEM and AEM for
Various Sample Sizes and Levels of Multicollinearity
\begin{tabular}{|c|l|l|c|}
\hline $\begin{array}{c}\text { Sample } \\
\text { Size (T) }\end{array}$ & $\begin{array}{l}\text { Cor(K, L) } \\
\mathbf{0 . 0 3}\end{array}$ & $\begin{array}{c}\text { Cor(K, L) } \\
\mathbf{0} \mathbf{0 . 2 4}\end{array}$ & $\begin{array}{c}\text { Cor(K, L) } \\
\mathbf{0}\end{array}$ \\
\hline 20 & $1.29 \mathrm{E} 08$ & $1.17 \mathrm{E} 08$ & $1.27 \mathrm{E} 08$ \\
\hline 40 & $1.61 \mathrm{E} 08$ & $0.74 \mathrm{E} 08$ & $1.37 \mathrm{E} 08$ \\
\hline 80 & $0.19 \mathrm{E} 08$ & $0.73 \mathrm{E} 08$ & $1.23 \mathrm{E} 08$ \\
\hline
\end{tabular}

The first model to be considered is:

$$
\text { AED / AEM : Y }=\theta_{1} K^{\theta_{2}} \mathrm{~L}^{\theta_{3}}+\mathrm{U}_{0}
$$

where $U_{0}$ follows $\mathrm{N}\left(0, \sigma_{0}^{2}\right)$. Monte Carlo results showing estimates of mean square error of $\hat{\wedge}^{2}$, $\operatorname{MSE}\left(\bar{R}^{2}\right)$ are presented in Table 1. In this table $\sigma_{0}^{2}=0.16, \theta=(10,0.45,0.50)$ with sample size $T=$ $20,40,80$ and replication $\mathrm{N}=20$.

Table 2 gives estimates for the model

$$
\text { MED / MEM : Y }=\theta_{1} \mathrm{~K}^{\theta_{2}} \mathrm{~L}^{\theta_{3}} \mathrm{e}^{\mathrm{U}_{0}} 4.2
$$

We still use $\sigma_{0}^{2}=0.16, \theta=(10,0.45,0.50), T=20$, 40,80 and $N=20$ to obtain required estimates.

Table 3 shows the impact of multicollinearity of the model MEM relative to the model AEM as sample size increases. Ratios of MSE in both models are used to achieve this.

\section{DISCUSSION}

One wonders why many decimal places are allowed in the computations. The outstanding reason is that while the $\operatorname{MSE}\left(\overline{\mathrm{R}}^{2}\right)$ values for two different models are small, the size of their ratios could be very high. For instance, when the level of collinearity between the inputs $\mathrm{K}$ and $\mathrm{L}$ is $0.03, \operatorname{MSE}\left(\hat{R}^{2}\right)$ values for MED/MEM and AED/AEM are respectively 0.046419 and $3.60 \mathrm{E}-10$ for $T=20$.. The ratio of these values is 1.29E08. This is high. (Please see Table 3)

The mean square error (MSE) of the estimated $\bar{R}^{2}$ gives the magnitude of the impact of presence of multicollinearity. The correlation coefficient 
Cor $(K, L)$,between the inputs $K$ and $L$ gives the level of multicollinearity between $\mathrm{K}$ and $\mathrm{L}$. Consider Table 1 and Table 2 ( Sample size $T=20$ ) and multicollinearity level $\operatorname{Cor}(K, L)=0.03$. The value of MSE in MEM is higher than MSE in AEM. The trend is the same for $\operatorname{Cor}(K, L)=0.24$ and $\operatorname{Cor}(K, L)=0.45$. When we go to higher sample sizes in Table 1 and Table 2, MEM still has higher MSE than AEM. In Table 3, the ratio of MSE in MEM to that in AEM is far greater than unity irrespective of sample size and level of multicollinearity.. Let us look at AEM and MEM as sample size increases for a given level of multicollinearity.

Take AEM first. When $\mathrm{Col}(\mathrm{K}, \mathrm{L})=0.03$,MSE decreases first and later rises and when $\operatorname{Col}(K, L)=$ 0.24 MSE decreases with sample size. Finally for $\mathrm{Col}(\mathrm{K}, \mathrm{L})=0.45$, MSE decreases and later rises. Here, taking all the three level of multicollinearity into consideration,there is no general trend for the behaviour of MSE as sample size increases. We observe that in MEM, when $\mathrm{Col}(\mathrm{K}, \mathrm{L})$ takes the values 0.24 and 0.45 , MSE decreases with sample size. MSE oscillate for $\operatorname{Col}(K, L)=0.03$. Again in three collinear cases, as was in the AEM, the trend of MSE as sample size increases is not well defined.

\section{CONCLUSION}

We, from the beginning do not focus on the detection of multicoliinearity as attempted by [ 9 ] but rather investigate the consequences and the seriousness of the consequences of the presence of multicollinearity. The adverse effect of multicollinearity in MEM is worse than the adverse effect of multicollinearity in AEM . The higher values of mean square error in MEM attest to this. However, as the sample size increases, the trend of the impact of multicollinearity is ambiguous for both AEM and MEM. This make us conclude that effect of multicollinearity cannot be said to have been purged by large sample size.

\section{REFERENCES}

[1] Dhyrmes, P.J. et al. 1972,"Criteria for Evaluation of Econometric Models , "Annals of Economic and Social Measurement, 1, 292-324.

[2] Essi, I. D., 2002 "Econometric Models with Mis-specified Error Terms ," Abacus: Journal of the Mathematical Association of Nigeria), Vol 29 (2), 152- 160

[3] Essi I D. 2005, "On Constructing Leaf Rectangularity Index by Bootstrap Regression ," AMSE Journal, Vol. 66 ( 6) , $13-22$.
[4] Essi, I.D. and lyaniwura J. O.,2007, "On Robustness and Choosing Between Two Nonlinearities ," Advances and Applications in Statistics7(3),451-462

[5] Essi I.D.,.(2007) Computing Leaf Regularity Index under Alternative Error Specifications ,AMSE Journal of Modeling C Vol. 70(1), 67 -79

[6] Essi, I.D , Iyaniwura, J.O. and Amadi S.N. ,2007 "Further on Monte Carlo Simulation of Inputs in Production Theory" AMSE Journal of Modeling, Vol 28(2), 23-35

[7] Essi, I.D., Iyaniwura, J.O. and Ojekudo N. A. ,2007 “On Multicollinearity in Nonlinear Econometric Models with Mis-specified Error Terms in Small Samples" Forthcoming in International Journal of Statistics and Systems(IJSS) Vol. 2(1),41-48

[8] Essi, N.O., 2000, "Robustness of Estimators of Nonlinear Econometric Models with Mis-specified Error Terms "- A Ph.D Thesis, University of Ibadan, Ibadan.

[9] Fabrycy, M. Z., 1975, " Multicollinearity Caused by Specification Errors," Applied Statistics, 24 (1), 250 260.

[10] Greene, W. H., 2003 ,"Econometric Analysis," $5^{\text {th }}$ ed. Prentice- Hall Englewood Cliffs, N.J.

[11] Heben, Julia, 1983, "Application of Econometrics," ,Oxford ,Philip Allen, p25

[12] Heben, Julia, 1983, "Application of Econometrics," Oxford, Philip Allen, pp90-98

[13] Theil, H., 1957, " Specification Errors and Estimation of Economic Relationships Review of the International Statistics Institute, " 25, 41 - 51

[14] Zarembka, P. ,1966, "Manufacturing and Agricultural Production Functions in International Trade". Journal of Economics, 47, 952-959.

[15] Zarembka, P. (1974).“Transformation of Variables in Econometrics", In Frontiers in Econometrics. Edited by P. Zarembka, New York, Academic Press, 81-104 